\title{
Reform and Exploration of Building Structure Experiment Teaching
}

\author{
Shaochong Yang, Jianhui Zhang \\ College of Civil Engineering \& Architecture, Hebei University, Baoding, Hebei, China \\ (yangshaochong@hbu.edu.cn), (hbuzjh@hbu.edu.cn)
}

\begin{abstract}
Based on the practice of the building structure experiment teaching, the present situation and existing problems of the teaching course were analyzed from the teaching idea, the teaching content and the teaching methods. The construction of the independent experimental teaching mode in the experiment course was explored, and was tried in the teaching practice. The years of teaching practice shows that the experimental teaching model is in favor of cultivating the student's manipulative ability, innovation ability, and suitable to the requirements of training innovative talents.
\end{abstract}

Key words: Building structure experiment, Teaching reform, Independent experiment teaching, Innovation ability training

\section{“建筑结构试验”课程教学改革与探索}

\author{
杨少冲 张建辉 \\ 河北大学 建筑工程学院, 保定, 河北, 中国
}

摘 要 基于建筑结构试验课教学的实际情况, 从教学理念、教学内容和教学方法等方面对本试验课程的教学现状及存在的问题 进行了分析; 对建筑结构试验课程独立实验教学模式的构建进行了探索, 并在教学实践中进行了尝试。多年的教学实践表明, 该教学 模式有利于学生动手能力、创新能力的培养, 适合创新型人才的培养要求。

关键词 建筑结构试验, 教学改革, 独立实验教学, 创新能力培养

1. 引言

建筑结构试验是一门面向结构工程检测和监测的基础 课程, 是土建类专业整个教学体系的重要组成部分, 是一 门旨在培养学生实践能力、创新能力的试验课程。其教学 任务是使学生获得并掌握工程结构试验方面的基础知识和 基本技能, 并能进行一般工程结构试验的规划和方案设计。 通过本课程的学习, 学生得到初步的训练和实践, 能在毕 业后更好地适应工作环境 [1-2]。随着结构试验设备和技术 的不断更新和发展, 原有的建筑结构试验课程教学模式已 不适应现在的教学要求。因此, 必须深化建筑结构试验课 程改革, 构建独立的实验教学模式。深化本课程的教学模 式改革、提高教学质量, 对于培养具有较强实践能力和创 新能力的人才具有重要意义 [3-6]。本文针对建筑结构试验 课程的教学理念、内容和方法等的改革进行了探讨, 提出

河北大学研究生教育教学改革项目支持 (资助号: YJ11-09)
了一些改革思路和措施, 并对适合培养创新型人才要求的 试验课程教学模式进行了尝试。旨在强化课程设计和实验 教学要求, 夯实学生应具备的基础理论和实验技能, 加强 案例教学和实践教学, 提高学生综合分析和动手解决问题 的能力。

\section{2. 建筑结构试验课程的教学现状及存在的主要问题 \\ 目前大多数高校土建类专业的建筑结构试验课程独立} 设课 [7], 课程包括理论和试验两部分教学内容, 经过多年 的教学实践, 已形成了一定的模式。但随着结构试验设备 和技术的不断更新、发展, 在本课程的教学工作中, 出现 了一些新问题, 主要有以下几个方面:

（1）教学理念上, 建筑结构试验课独立的实验教学环 节相对缺乏, 实验教学多依附于理论教学; 重理论、轻实 验, 重课堂教学、轻实验教学的传统教育观念仍然根深蒂 固, 实验课时偏少, 实验课程往往被看作是次要课程和理论 
课程的附属品。

（2）教学内容上, 建筑结构试验教学内容过分注重对 实验仪器、实验步骤的细节性描述, 较少有实验思想、设 计思路、实验研究等素质教育的内容; 一些研究性、设计 性实验因实验教学学时少和实验条件的限制几乎无法实

施, 学生被束缚在规定的实验框架中; 单纯验证、演示性 实验往往使学生感到乏味, 难以激发学生对实验的浓厚兴 趣和探索精神, 更难以培养学生观察、发现、创新地分析 问题和解决问题的能力, 不利于学生能力的培养。

（3）教学方法上, 结构试验课程通常采用刻板的、填 鸭式演示实验为主, 学生在教师示范下进行实验或根据实 验教材中所述步骤按部就班做实验, 不利于学生独立思考、 以及解决实验过程中可能遇到的问题; 造成学生不能熟练 掌握实验操作技能, 不会分析处理实验数据和编写报告, 缺乏解决综合性问题的能力和相互协作的团队精神。这种 教学方法培养出来的学生虽然可能具有一定的专业知识, 但学生往往动手能力低下, 缺乏科学分析和解决问题的能 力。

因此, 有必要进一步深化建筑结构试验课程改革, 构 建独立的实验教学模式, 充分发挥学生的学习积极性和主 观能动性, 提高学生实验设计与实施的综合能力。

\section{3. 建筑结构试验课程独立实验教学模式的构建}

构建建筑结构试验课程独立的实验教学模式是近几年 实验教学改革的发展趋势, 是为适应社会发展需要, 满足 学生创新能力培养要求而进行的重要改革。近年来, 我校 在建筑结构试验课程的教学实践中, 对构建实验教学与理 论教学并重的突出综合性、设计性、创新性的实验教学模 式, 进行了有益的尝试。

(1) 改变以演示性试验为主的教学局面, 增设设计性、 综合性以及研究性的实验项目 [8-12]。课程中实验项目主 要以设计性、综合性实验为主, 让学生充分参与实验的设 计和实验教学全过程, 做到人人参与, 人人动手, 保证学 会必要的实验方法, 强化掌握试验基本技能; 及时向同学 传授当前先进仪器设备的功能、测量方法、分析软件的使 用及可靠性程度等要素, 正确分析实验结果、处理数据, 培养学生严肃的科学态度和严谨的科学作风。同时, 在实 验教学中, 注重巩固学生的理论知识, 设计研究性教学环 节, 渗透研究性教学理念, 充分发挥学生的学习主动性, 引导学生开展自主、开放、探究、合作式的研究性学习, 培养学生的独立实验能力和启发学生的创新灵感。这样学 生可以综合运用所学的知识, 活跃思维, 培养全面技能, 并能开发学生的创造力和独立分析与解决问题的能力。
(2) 采用以学生为主体、教师引导和师生共同研讨为 主的互动式教学方法。在教学过程中, 强调以学生为主体, 师生互动, 克服传统的简单说教式教学方法的弊端。充分 发挥教师知识传授者、学生学习的引导者与合作者的作用, 充分调动学生的主观能动性, 强调自主学习和自主研究的 教学方式。在教师引导下, 做到学生自己查资料、看仪器、 做实验、提问题、解疑难, 培养学生发现问题、分析问题 和解决问题的能力。强调学生的独立性、主动性和学习积 极性, 学生不再是单纯的实验操作者, 而是实验构思和设 计的主导者以及实验过程的控制者, 并由此培养学生的创 新意识和能力。

(3) 利用产学研结合, 提升实验教学质量 [13-15]。 将学院实验教学中心承接的纵向、横向科研项目作为建筑 结构试验课程教学的重要平台。让学生参与实际工程的测 试、分析, 以及相关科研项目的研究工作。将工程实例引 入实验教学中, 将实际结构测试项目与实验教学紧密结合, 通过这种实战性实验教学, 提高学生解决实际问题的能力。 帮助学生消除对实际工程结构试验、测试的神秘感, 有效 地培养学生的创新精神和分析、解决实际问题的能力。例 如让我校学生参与某桥梁检测项目, 根据检测要求, 设计 试验测试方案, 现场实际操作应变片、挠度计的安装和测 试仪器的连接, 掌握应变、挠度等测试项目的测量方法, 正确进行数据处理与数据分析, 以及撰写检测报告等。这 样, 使学生全面参与工程结构测试的全过程, 并掌握了包 括实验准备、设计、实施、及结果数据分析的方法, 获得 了在课堂上难以达到的良好教学效果, 受到同学们的欢迎。

(4) 为了达到预期的实验目的, 要求学生必须注意以 下几方面问题

试验前认真预习实验指导书、课本有关内容及实验设 备使用说明, 充分了解各个试验的目的、试验原理、方法 和步骤, 并进行一些必要的理论计算以便与实验数据进行 比较。

较大的综合试验, 应选出一名小组长, 负责组织和指 挥整个试验过程, 直至把处理好的试验数据及试验报告完 成, 小组各成员必须服从小组长和指导教师的指挥, 要明 确分工, 不得撤离各自的岗位。

试验时应严肃认真, 密切注意观察试验现象, 及时加 以分析和记录, 试验报告要求原始记录齐全、计算分析正 确、数据图表清楚。每位同学应独立完成试验报告, 除小 组分工由别人记录的原始数据外, 严禁抄袭。

\section{4. 主要实验教学环节的设计}

为了达到上述所构建的独立实验教学模式要求, 根据 
我校现有专业设置和试验设备把建筑结构试验分为应变及 位移测量、超声回弹综合法检测混凝土强度和模态测试三 个大的综合实验。

\section{1 应变及位移等物理量的测量}

电阻应变测量依据电阻丝的电阻率随电阻丝的变形而 变化的关系, 把力学参数转换成与之成比例的电学参数, 通 过测量电学参数并依照一定的比例关系将其转换成试件的 应变值 (位移值)。正确掌握其测量方法是较好地进行实验 应力分析的基础。通过试验让学生熟悉电阻应变测量工作 原理, 根据实际测量需求确定电桥的连接方法, 并指出测 量过程中产生误差的原因及控制误差的方法。

(1) 应变测量方案设计。首先要根据测量目的选取测 点的位置, 选择测点的位置还要根据受载构件应力分布, 在应力较大或位置特殊处布置应变片传感器。然后根据构 件尺寸、材料、测量精度要求和应力梯度来选择应变片的 栅长和型号。测量导线的布置应整齐, 避开电磁场的干扰, 接线后要检查编号、绝缘电阻和应变计阻值, 同时还要进 行防潮处理。

(2) 由于温度和湿度的变化, 大多数敏感材料的灵敏 系数是随着工作温度的升高和湿度的增大而不断减小。粘 结剂和基底材料传递应变的能力随工作温度的升高也逐渐 下降。所以工作温度越高, 湿度越大, 灵敏系数 $K$ 值的下降 就越快, 另外, 电阻丝受力后其电阻率也会发生变化, 从而 引起 $K$ 值的变化。所以在测量时应尽可能保持标定灵敏系数 $K$ 时的工作环境, 从而减小由于工作环境的变化所产生的测 量误差。

(3) 应变计的粘贴方向如果与测点要求的方向不重 合, 则会给测量结果带来误差, 在粘贴应变计时, 尽可能 减小方向偏差, 采用同一基底上有多轴敏感栅的应力花有 助于提高粘贴方向的准确性。在测量中, 由于构件尺寸很 大, 同时出于安全考虑, 测量仪器往往都需要与构件有一 定的距离, 因此需要很长的导线连接应变计和测量仪器, 但这样就使得导线分布电容变大, 对应变量的结果有一定 的影响, 在最后计算时, 必须按不同桥路接法进行修正, 在此不详细列举。

采用应变电测的方法具有精度高、应用方便等诸多优 点。但是影响应变测量精度的因素有很多, 包括测量的设 计方案、应变计的粘贴工艺、应变计的布置与桥路连接方 法等诸多因素。因此, 在测量时对诸多因素进行综合分析 和全面修正是保证精确测量的关键。

4.2 超声回弹综合法检测混凝土强度

回弹法检测混凝土强度因其测试简便、快速等特点在 工程质量监督检测中得到广泛应用。但其测试精度易受含
水率、碳化深度等表面状态的影响。混凝土的强度与波速 $\mathrm{v}$ 、 混凝土回弹值 $\mathrm{R}$ 之间有较好的相关性, 强度越高, 波速越快, 回弹值越高。超声回弹综合法检测混凝土强度的优点如下:

(1) 回弹法通过混凝土表层的弹性和硬度反映混凝土 的强度, 超声波通过整个截面的弹性反映混凝土的强度。 回弹法测试低强度混凝土时, 由于弹击可能产生较大的塑 性变形影响测试精度, 而超声波的声速随混凝土强度增长 到一定程度后, 增长速度下降, 因此, 超声对较高强度混 凝土不敏感。采用超声回弹法可以内外结合, 相互弥补, 较全面的反映混凝土的实际质量。

（2）混凝土的龄期和含水率对回弹值和声速都有影 响, 混凝土含水率大, 超声波的声速偏高, 而回弹值偏低; 另一方面, 混凝土的龄期长, 回弹因混凝土表面碳化深度 增加而增加, 但超声波的声速随龄期增加的幅度有限, 两 者结合的综合法可减少混凝土龄期和含水率的影响。

\section{3 模态测试}

模态分析方法是把复杂的实际结构简化成模态模型, 来进行系统的参数识别 (系统识别) , 从而大大地简化了 系统的数学运算。

（1）模态分析用于振动测量和结构动力学分析。可测 得比较精确的固有频率、模态振型、模态阻尼、模态质量 和模态刚度; 可用模态实验结果去指导有限元理论模型的 修正, 使计算机模型更趋于完善和合理; 用来进行结构动 力学修改、灵敏度分析和反问题的计算; 用来进行响应计 算和载荷识别。

（2）模态分析基本原理。采用简化的方法, 把结构归 结为有限个自由度的模型来进行分析, 即将系统抽象为由 一些集中质量块和弹性元件组成的模型。模态分析的实质, 是一种坐标转换。其目的在于把原在物理坐标系统中描述 的响应向量, 放到所谓 “模态坐标系统” 中来描述。这一 坐标系统的每一个基向量恰是振动系统的一个特征向量。 也就是说在这个坐标下, 振动方程是一组互无耦合的方程, 分别描述振动系统的各阶振动形式, 每个坐标均可单独求 解, 得到系统的某阶结构参数。

(3) 模态测试过程。为进行模态分析, 首先要测得激 振力及相应的响应信号, 进行传递函数分析。然后建立结 构模型, 采用适当的方法进行模态拟合, 得到各阶模态参 数和相应的模态振型, 形象地描述出系统的振动型态。实 际应用时, 单击拾振法, 常用锤击法激振, 用于结构较为 轻小, 阻尼不大的情况。对于笨重、大型及阻尼较大的系 统, 则常用固定点激振的方法, 用激振器激励, 以提供足 够的能量。锤击法简支梁模态测试可以选用单点拾振法 (跑 
激励), 也可以选用单点激励法 (跑响应); 而随机激励法模 态实验由于移动激励比较困难, 工作量大, 所以一般情况 下多采用单点激励法。总的来说, 随机激励法和锤击法简 支梁模态测试方法在原理上是基本相同的。

\section{5. 结束语}

建筑结构试验教学改革是一项复杂而细致的工作, 是 一个不断完善并发展的过程。多年的教学实践表明, 注重 理论与实验教学并重, 强调实践环节, 通过综合性、研究 性实验的实施, 全面训练学生对建筑结构试验基本理论的 应用能力; 同时通过对实践性环节的综合设计, 灵活教与 学的关系, 使学生主动参与、自主完成从实验设计、试件 制作、加载测试、实验结果分析和总结的全过程, 这种探 索很有成效。本文所进行的建筑结构试验课独立的实验教 学模式的尝试, 对于培养学生的动手能力、综合分析能力、 独立解决问题的能力取得良好效果, 深受学生欢迎, 达到 了提升学生综合能力与素质、培养学生创新意识与能力的 教学目标。

\section{参考文献(References)}

[1] Yi Weijian, Zhang Wangxi. Architectural Structure Experimentation. Beijing: China Construction Industry Press, 2005.

[2] Xiong Zhongming, Wang Duliang. Civil engineering experiment and test. Beijing: China Construction Industry Press, 2007.

[3] Zeng Lei, Xu Chengxiang, Liu Changming. Reform and Exploration of Experimental Teaching System in Civil Engineering. Journal of Architectural Education In Institutions of Higher Learning, 2009, 18(4):119-122.

[4] Yang Yiping, Li Ping, Wang Guixue, Qin Jian. Discussion on the Experimental Teaching Model of Fostering the Students with Innovative Auality. Research and Exploration in Laboratory, 2011,30(7):259-261

[5] Li Li, Xiong Shishu. Experiment Teaching System and Experiment Project Innovation of C ivil Engineering. Journal of
Architectural Education in Institutions of Higher Learning, 2010, 19(6):141-143.

[6] Lin Jianhao,Yue Hang. Reform of Civil Engineering Materials Experimental Teaching. Journal of Tianzhong, 2010, 25(2): 100-102.

[7] Huang Dam ing, Qin Gangnian, Wen Bing, Liang Junbo. Study and Practice of the Independent Curriculum of Professional Experiment. Research and Exploration in Laboratory, 2007, 26(11):85-94.

[8] Li Guangwei, Hu Liuxian. Exploration and Practice on Reform of Experimental Teaching and Training of Creative Talents. Research and Exploration in Laboratory, 2008, 27(9):100-103.

[9] Lu Wensheng, Huang B aofeng. Research and Practice of Experimental Teaching of Structural Seismic Methodology. Research and Exploration in Laboratory, 2009, 28(3):217-223.

[10] Wang Shaogang, Liu Renp ei, Feng Xiaosong. Developing Comprehensive Experiment to Cultivate Innovation Ability. Research and Exploration in Laboratory, 2009, 28(9):117-148.

[11] Liang Xilong, Fang Shumei. Thoughts on Higher comprehensive design experiment teaching. Higher Education of Sciences, 2007(5):125-127.

[12] Huang Daming, Qin Gangnian. Innovative Design and Teaching Practice of Comprehensive Experiments in Professional Experimental Course. Research and Exploration in Laboratory, 2011, 30(6):247-250.

[13] Wei Hua, Zeng Dongmei2, Qin Gangnian. Combining experimental teaching with scientific research to develop students innovative ability. Experimental Technology and Management, 2008, 25(5):31-34.

[14] Cao Jiangzhong, Dai Qingyun, He Jiafeng. Exploration of the Reform of Experimental Teaching in Cooperation of Production, Teaching and Research. Research and Exploration in Laboratory, 2011,30(6):287-290

[15]Zhou Yan, Gao Yinhan, Jin Wei, Meng Fanyu. Exploration of the Innovative Ability Training by the Indus try University Research (IUR) Combination. Research and Exploration in Laboratory, 2009, 28(5):5-7. 